\title{
Scalable Nanomanufacturing of Nanoparticle Films: A Continuous Automated Langmuir-Blodgett Assembly and Deposition Method
}

\section{Xue Li*}

Department of Chemical and Biomolecular Engineering, Johns Hopkins University, Baltimore, Maryland 21218, USA

Nanoparticles (NP) of different materials keep capturing attention of researchers for several reasons. First, they may have properties that differ from the bulk material. Even if that is not the case, producing materials in nanoparticle form (especially by solution methods) can be advantageous if synthesis of bulk material is complicated and/or costly. Irrespective of the motivation behind making the nanoparticles, Nanoparticle in the form of thin film has attracted ever-increasing attention due to its practical application and scientific importance in numerous fields such as nanodevices [1], electronics [2], functional coatings [3]. Therefore, a great number of publications have studied the deposition of nanoparticle thin film.

Most commonly used techniques include: Langmuir-Blodgett (LB) technique [4], dip coating, spin coating, colloidal evaporation, electrostatic adsorption, chemical vapour deposition (CVD). The most common processes for fabrication of colloidal monolayers and colloidal crystals is using Langmuir-Blodgett deposition, convective deposition, and spin coating. Langmuir-Blodgett deposition [5,6] is well known for its ability to transfer amphiphilic molecules from a stagnant liquid interface onto a solid substrate, and has been extended to transfer other macromolecules and particles trapped at an air-liquid interface. While this process can be scaled to large troughs, it is not particularly continuous and the rate of deposition must be coordinated with the surface pressure by moving one or more walls of the trough. Convective deposition, which takes advantage of the evaporative flux of the solvent, can be scaled but the rate of deposition and assembly is highly limited by the solvent properties. Likewise, larger scale coatings can succumb to streaks formed by local instabilities. These streaks can be inhibited by using binary colloidal suspensions [7] or vibration-assisted convective deposition [8]. For monolayer deposition, convective deposition is limited to tens of microns per second. Peng and coworkers [9] have demonstrated fabrication of larger scale colloidal crystals using spin coating. However, the area coated is limited to the size of the wafer and cannot be translated to a roll-to-roll platform.

Most recently, Chokprasombat et al. [10] claimed preparation of long-range magnetic particles monolayers, the largest area they obtained is about $3 \mathrm{~mm}^{2}$. Xia [11] successfully created macroscopic freestanding close-packed gold nanoparticle monolayer film by water/ alcohol interfacial self-assembly, but their films are not larger than 1 $\mathrm{cm}^{2}$. A even Larger area of $200 \mathrm{~cm}^{2}$ hydrophilic citrate-stabilized $\mathrm{Au}$ nanoparticle monolayer films has been reported but not continuous process [12]. Although these researches use well-established techniques to fabricate nanoparticle thin films over a relatively large area, they are still limiting their potential capability of even larger area of nanoparticle films to industrial scale continuously. There are significant challenges to fabricating coatings that have well-defined 2D and 3D nano- and meso-scale structure through continuous, commercial scale processes.

Automated Langmuir-Blodgett (ALB) deposition, was first developed by researchers at University of Toronto [13], allowing a facile and general way to fabricate macroscopic closely packed-alcohol nanoparticle interfaces films on solid substrate at water in a continuous manner over a large area. By using such technique, one can tailor the advantage of LB for assembling high-density nanoparticle monolayers and multilayer films and the easy speed manipulation of dip coating method. And thus yielding a scaled up process of nanoparticle film depositon to large areas $(10 \mathrm{~cm} \times$ desired length film of depositionthe to large areas $(10 \mathrm{~cm}$ substrate roll) with fast speed $(1-100 \mathrm{~mm} / \mathrm{sec})$, comparable with industrial standard [14]. The assembled nanoparticle layers (Langmuir films) can be transferred to arbitrary substrates either flexible or rigid ones. Furthermore, the morphology of the Langmuir film can be effectively controlled by adjusting parameters such as: the particles concentration and web speed.

The automated Langmuir-Blodgett technique offers a facial and general way to fabricate macroscopic, dried, closely-packed nanoparticles films on solid substrate at liquid/alcohol interfaces over a large area. Due to its independence of the particles chemical nature, this approach can be applied to a large variety of particles and surfactants for the formation of macroscopic particle films. And thus it is a "scalable nanomanufacturing" which has promising practical industrial application including electronics, biomedical devices, food science, water treatment, and smart packaging.

\section{References}

1. Liu S, Akbashev AR, Yang X, Liu X, Li W, et al. (2014) Hollandites as a New Class of Multiferroics. Sci Rep.

2. Kong YL, Tamargo IA, Kim H, Johnson BN, Gupta MK, et al. (2014) 3D Printed Quantum Dot Light-Emitting Diodes. Nano Lett 14: 7017-7023.

3. Ge J, Zeng X, Tao X, Li X, Shen Z, et al. (2010) Preparation and Characterization of PS-PMMA/ZnO Nanocomposite Films with Novel Properties of High Transparency and UV-Shielding Capacity. J Appl Polym Sci 118: 1507-1512.

4. Zheng Q, Zhang B, Lin X, Shen X, Yousefi N, et al. (2012) Highly Transparent and Conducting Ultralarge Graphene Oxide/single-Walled Carbon Nanotube Hybrid Films Produced by Langmuir-Blodgett Assembly. J Mater Chem 22: 25072.

5. Kumar V, Bahadur N, Sachdev D, Gupta S, Reddy GB, et al. (2014) Restructural Confirmation and Photocatalytic Applications of Graphene Oxidegold Composites Synthesized by Langmuir-Blodgett Method. Carbon N Y 80: 290-304.

6. Modlińska A, Bauman D (2011) The Langmuir -Blodgett Technique as a Too for Homeotropic Alignment of Fluorinated Liquid Crystals Mixed with Arachidic Acid. Int J Mol Sci 12: 4923-4945.

7. Kumnorkaew P, Weldon AL, Gilchrist JF (2010) Matching Constituent Fluxes for Convective Deposition of Binary Suspensions. Langmuir 26: 2401-2405.

*Corresponding author: Xue $\mathrm{Li}$, Department of Chemical and Biomolecula Engineering, Johns Hopkins University, Baltimore, Maryland 21218, USA, Tel: +1 4105168000; E-mail: xueli2014x@gmail.com

Received July 07, 2016; Accepted July 07, 2016; Published July 14, 2016

Citation: Li X (2016) Scalable Nanomanufacturing of Nanoparticle Films: A Continuous Automated Langmuir-Blodgett Assembly and Deposition Method. J Electr Electron Syst 5: e116. doi: 10.4172/2332-0796.1000e116

Copyright: ( ) $2016 \mathrm{Li} \mathrm{X}$. This is an open-access article distributed under the terms of the Creative Commons Attribution License, which permits unrestricted use, distribution, and reproduction in any medium, provided the original author and source are credited. 
Citation: Li X (2016) Scalable Nanomanufacturing of Nanoparticle Films: A Continuous Automated Langmuir-Blodgett Assembly and Deposition Method. J Electr Electron Syst 5: e116. doi: 10.4172/2332-0796.1000e116

Page 2 of 2

8. Muangnapoh T, Weldon AL, Gilchrist JF (2013) Enhanced Colloidal Monolayer Assembly via Vibration-Assisted Convective Deposition. Appl Phys Lett.

9. Jiang P, McFarland MJ (2004) Large-Scale Fabrication of Wafer-Size Colloidal Crystals, Macroporous Polymers and Nanocomposites by Spin-Coating. J Am Chem Soc 126: 13778-13786.

10. Chokprasombat K, Sirisathitkul C, Ratphonsan P (2014) Liquid-Air Interface Self-Assembly: A Facile Method to Fabricate Long-Range Nanoparticle Monolayers. Surf Sci 621: 162-167.

11. Xia H, Wang D (2008) Fabrication of Macroscopic Freestanding Films of
Metallic Nanoparticle Monolayers by Interfacial Self-Assembly. Adv Mater 20: 4253-4256.

12. Liu C, Li YJ, Wang MH, He Y, Yeung ES (2009) Rapid Fabrication of LargeArea Nanoparticle Monolayer Films via Water-Induced Interfacial Assembly. Nanotechnology.

13. Schneider J (2010) Method and Apparatus for Thin Film/layer Fabrication and Deposition.

14. Li X, Gilchrist JF (2016) Large-Area Nanoparticle Films by Continuous Automated Langmuir-Blodgett Assembly and Deposition. Langmuir 32: 1220-1226. 